\title{
Fratura proximal do fêmur e lesão vascular em adultos - Relato de caso*
}

\section{Proximal Femur Fracture and Vascular Injury in Adults - Case Report}

\author{
Pedro José Labronici ${ }^{1,2}$ Fernando Claudino dos Santos Filho ${ }^{1}$ Yuri Leander Oliveira Diamantino ${ }^{1}$ \\ Eduardo Loureiro $^{3,4}$ Maria Cristina Diniz Gonçalves Ezequiel ${ }^{2}$ Sérgio Delmonte Alves ${ }^{5}$
}

${ }^{1}$ Serviço de Ortopedia e Traumatologia, Hospital Santa Teresa, Petrópolis, RJ, Brasil

2 Universidade Federal Fluminense, Niterói, RJ, Brasil

${ }^{3}$ Cirurgia Vascular, Hospital Santa Teresa, Petrópolis, RJ, Brasil

${ }^{4}$ Cirurgia Vascular, Hospital Federal da Lagoa, Rio de Janeiro, RJ, Brasil

${ }^{5}$ Serviço de Trauma, Hospital Escola São Vicente Paula, Passo Fundo, RS, Brasil

Rev Bras Ortop 2019;54:343-346.

\begin{abstract}
Address for correspondence Pedro José Labronici, PhD, Serviço de Ortopedia e Traumatologia, Hospital Santa Teresa, Petrópolis, RJ, Brasil (e-mail: plabronici@globo.com).
\end{abstract}

\section{Resumo \\ Palavras-chave \\ - fraturas do fêmur \\ - lesões do sistema vascular \\ - parafusos ósseos \\ - quadril/cirurgia}

Complicações vasculares no tratamento cirúrgico da fratura do quadril são raras. A depender da lesão arterial, pode ocorrer um grave sangramento intraoperatório ou formação de hematoma subagudo com desenvolvimento de pseudoaneurisma arterial. Na literatura, as complicações mais frequentes relatadas são a formação de grandes hematomas locais após osteossíntese com parafuso deslizante do quadril. O objetivo do presente relato foi demonstrar um caso de lesão arterial tardia após osteossíntese proximal do fêmur.

Vascular complications in the surgical treatment of hip fractures are rare. Depending on the arterial injury, severe intraoperative bleeding can occur, or formation of a subacute hematoma with development of arterial pseudoaneurysm. In the literature, the more frequently described complications are the formation of local large hematoma after osteosynthesis with sliding hip screw. The objective of the present report was to demonstrate a case of delayed arterial injury after proximal femur osteosynthesis.

\section{Introdução}

A taxa de complicações vasculares no tratamento cirúrgico da fratura do quadril é baixa, com uma incidência de $0,2 \%{ }^{1} \mathrm{O}$

\footnotetext{
Trabalho desenvolvido no Serviço de Ortopedia e Traumatologia, Hospital Santa Teresa, Petrópolis, RJ, Brasil. Publicado originalmente por Elsevier Ltda.

(1) Pedro José Labronici's ORCID is https://orcid.org/0000-0003-49677576.
}

received

August 16, 2017

accepted

September 19, 2017
DOI https://doi.org/

10.1016/j.rbo.2017.09.003. ISSN 0102-3616. comprometimento vascular nesta região anatômica pode ser causado por fragmentos ósseos, relacionados a razões iatrogênicas, parafusos de bloqueio das hastes intramedulares, mau posicionamento dos afastadores, perfuração com brocas ou movimentação dos fragmentos ósseos durante a redução. ${ }^{2} \mathrm{~A}$ depender do defeito na parede do vaso, a lesão pode causar um maciço sangramento intraoperatório ou formação de hematoma subagudo com desenvolvimento de pseudoaneurisma arterial. Vários autores observaram altas taxas de comprometimento vascular oculto após o uso de placas proximais no
Copyright $(2019$ by Sociedade Brasileira License terms de Ortopedia e Traumatologia. Published by Thieme Revnter Publicações Ltda, Rio de Janeiro, Brazil 

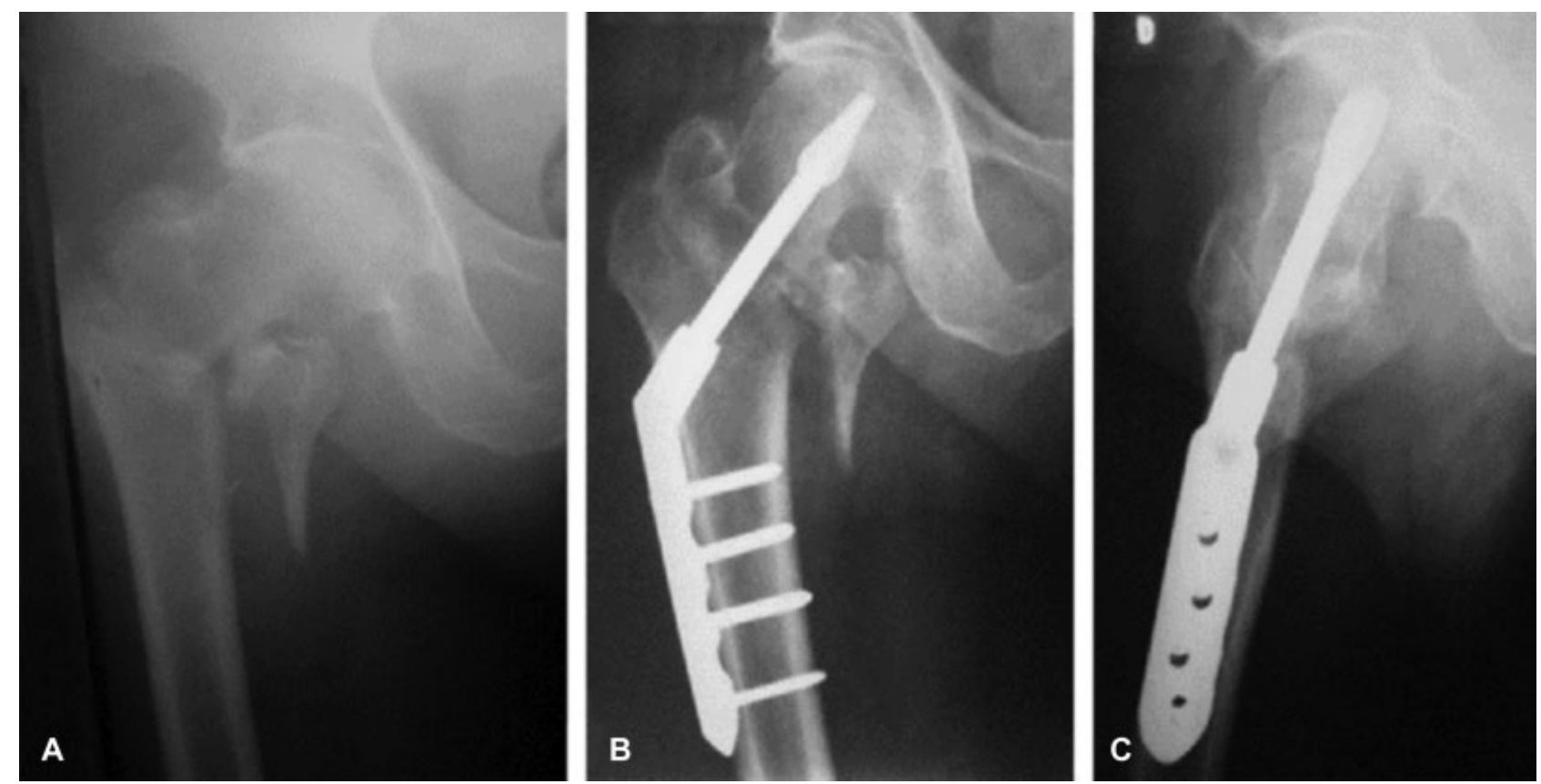

Fig. 1 A, radiografia da fratura transtrocantérica à direita; B e C, fratura tratada com parafuso deslizante do quadril (DHHS).

fêmur. ${ }^{3} \mathrm{Na}$ literatura, as complicações mais frequentes relatadas são a formação de grandes hematomas locais após osteossíntese com parafuso deslizante do quadril. ${ }^{1-3}$

O objetivo do presente relato de caso é demonstrar um caso de lesão arterial tardia após osteossíntese proximal do fêmur.

\section{Relato de Caso}

Paciente do gênero masculino de 87 anos, branco, deu entrada na emergência, após 3 horas de queda, com dor, incapacidade funcional e rotação externa do membro inferior esquerdo. 0 exame radiográfico demonstrou fratura proximal do fêmur esquerdo, do tipo transtrocantérica e classificação AO como 31-A2. Relata diabetes mellitus tipo II, polineurite diabética, osteopenia e hipertensão arterial sistêmica.

O paciente foi tratado 24 horas após a queda com osteossíntese do tipo prego e placa deslizante do tipo DHHS Synthes (DePuy-Synthes companies of Johnson\&Johnson). O procedimento ocorreu sem intercorrências (-Fig. 1). Após 23 dias da cirurgia, o paciente apresentou dor súbita e impotência funcional importante do membro inferior esquerdo, com irradiação ao joelho ipsilateral e aumento do diâmetro da coxa $(55 \mathrm{~cm}$ para $58 \mathrm{~cm}) .0$ exame físico mostrava hipotensão arterial $(90 \times 60 \mathrm{mmHg}$ ), frequência cardíaca de $100 \mathrm{bpm}$, sudorese fria e palidez; hematócrito de 16\% e hemoglobina em $58 \%$.

O exame de ultrassonografia do quadril esquerdo demonstrou grande infiltração de partes moles com detritos na coxa, na perna e no quadrante inferior do abdome.

A arteriografia evidenciou lesão aneurismática, que se comunicava com o escape, na artéria femoral esquerda.

O cirurgião vascular optou pela colocação de dois stents na artéria femoral profunda e o paciente apresentou melhoria significativa do quadro clínico e alta hospitalar (-Fig. 2).
Seis meses após a última intervenção cirúrgica, o paciente relata queixa em queimação e dor aguda pulsátil em região medial da coxa e joelho esquerdo que piorava com decúbito dorsal e melhorava ao se levantar. Foi solicitada uma angiotomografia, que identificou uma espícula óssea do trocânter menor que transpassava a artéria femoral profunda ( - Fig. $3 \mathrm{~A}$ e B). O paciente foi submetido a uma nova intervenção cirúrgica para retirada de um fragmento do trocânter menor, que apresentava lesão na artéria femoral profunda, e sutura da artéria (-Figs. 4 e 5). Dois dias após a intervenção cirúrgica, o paciente teve alta sem maiores intercorrências.

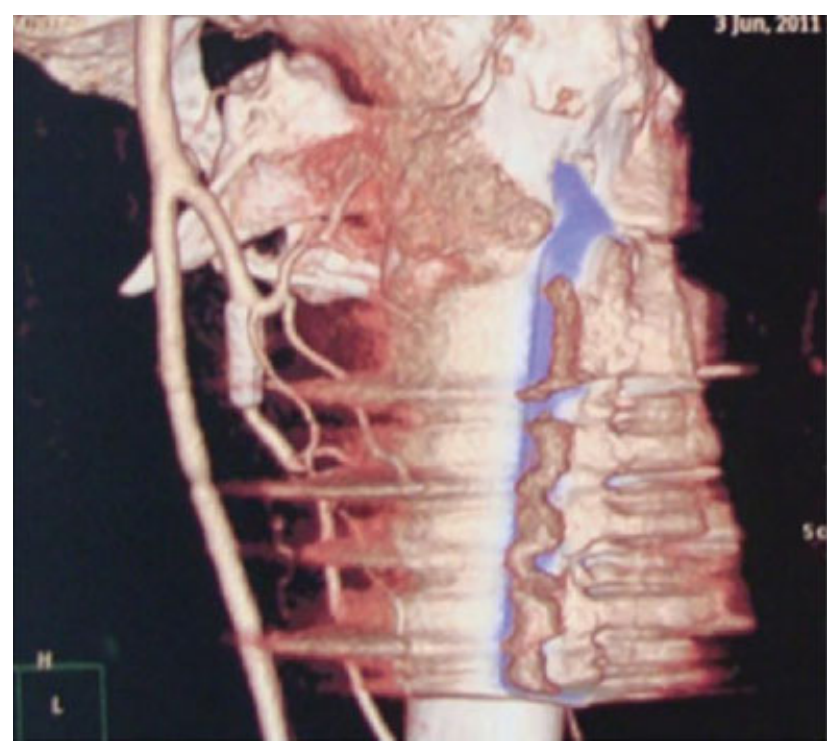

Fig. 2 Angiotomografia mostra o desvio do trocânter menor e artéria femoral profunda com stent. 

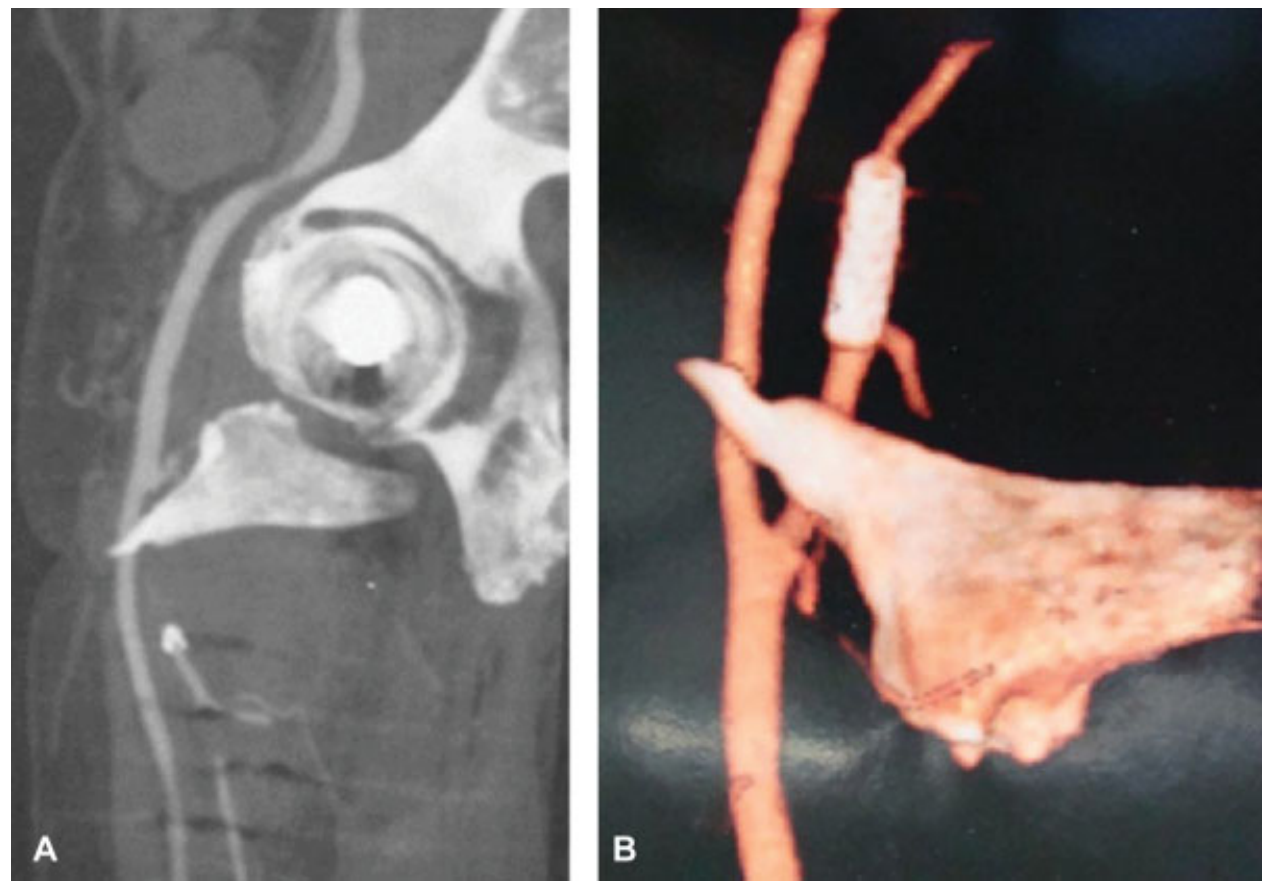

Fig. 3 A e B, angiotomografia mostra trocânter menor que perfura a artéria femoral profunda.

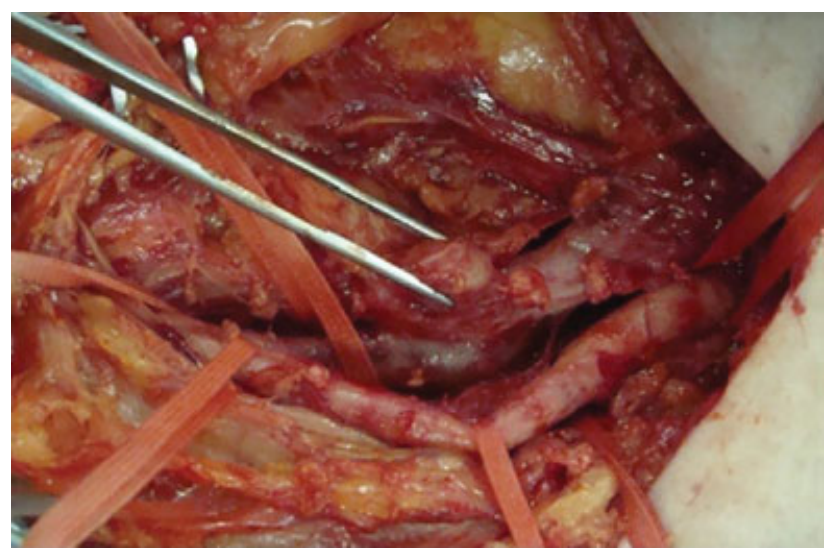

Fig. 4 Fotografia mostra pinça anatômica no pseudoaneurisma da artéria femoral profunda.

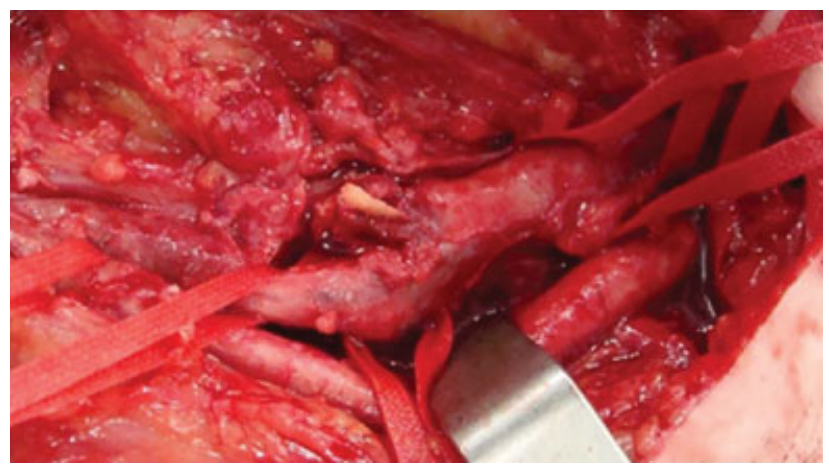

Fig. 5 Fotografia mostra espícula óssea do trocânter menor que transpassa a artéria femoral profunda.

\section{Discussão}

A incidência do comprometimento vascular após a estabilização da fratura do quadril é rara. Entretanto, quando essas complicações ocorrem, representam uma ameaça em potencial à função do membro ao longo do tempo ou podem mesmo gerar um resultado fatal.

Barquet et $\mathrm{al}^{4}$ demonstraram que a maioria das lesões vasculares é extrapélvica $(91,20 \%)$ em comparação com as intrapélvicas (8,24\%). No grupo de lesões extrapélvicas, houve uma alta prevalência de lesões da artéria femoral profunda e seus ramos em 78,31\%. Poucos casos ocorreram nos vasos da femoral superficial $(10,84 \%)$ e menos ainda que envolvessem outras artérias da coxa. Nosso paciente apresentou a lesão da artéria femoral profunda, a artéria mais comumente acometida.

Os tipos de lesão vascular incluem compressão com restrição do fluxo sanguíneo, lesão em flap da íntima com diminuição do fluxo distal, ruptura da camada íntima ou da placa de arteriosclerose com trombose arterial ou tromboembolismo, ligação cirúrgica que produz isquemia do membro, hemorragia aguda por laceração ou transecção e perfuração e progressiva erosão da artéria, que produz um pseudoaneurisma ou fístula arteriovenosa. 1,5,6 Existe uma alta prevalência para erosão da artéria, que produz um pseudoaneurisma. Nosso paciente passou por dois estágios de lesão arterial. 0 primeiro foi o da compressão com restrição do fluxo sanguíneo, no qual desenvolveu dor na região do quadril por um ano com edema de $>3 \mathrm{~cm}$ da coxa, e foi tratado com uma colocação de stent da artéria. Após esse período, desenvolveu um segundo estágio, apresentou dor aguda na região inguinal, 
com perfuração e progressiva erosão da artéria, e desenvolveu um pseudoaneurisma.

As lesões vasculares podem ser causadas por fragmentos da fratura, predominantemente pelo trocânter menor desviado. ${ }^{7}$ Barquet et $\mathrm{al}^{4}$ demonstraram sete lesões não iatrogênicas produzidas pelo desvio do trocânter menor ou outros fragmentos ósseos, tanto em pacientes com tratamento conservador ou após a fixação cirúrgica. Alguns autores preconizam monitoramento perioperatório do trocânter menor, tanto no desvio medial como no proximal, e pode ser necessária a redução, fixação ou remoção deste fragmento. ${ }^{8-10}$ Nosso paciente apresentou uma lesão da artéria femoral profunda tardiamente com formação de um pseudoaneurisma, causada pelo trocânter menor desviado, após um ano de fratura.

Lesões vasculares após fraturas proximais do fêmur acometem mais a artéria femoral profunda. Os pseudoaneurismas são mais frequentes e revelam um diagnóstico tardio da lesão. A lesão não iatrogênica mais frequente é a causada pelo desvio do fragmento do trocânter menor.

Conflitos de interesses

Os autores declaram não haver conflitos de interesses.

\section{Referências}

1 Karanikas I, Lazarides M, Arvanitis D, Papayanopoulos G, Exarchou E, Dayantas J. Iatrogenic arterial trauma associated with hip fracture surgery. Acta Chir Belg 1993;93(06):284-286
2 Klinger HM, Baums MH, Eckert M, Neugebauer R. [A comparative study of unstable per- and intertrochanteric femoral fractures treated with dynamic hip screw (DHS) and trochanteric buttpress plate vs. proximal femoral nail (PFN)]. Zentralbl Chir 2005; 130(04):301-306

3 Garrido-Gómez J, Garrido-Gómez MN, Arrabal-Polo MA, GarridoPareja F, Linares-Palomino JP. Iatrogenic false aneurysms. A rare complication of hip surgery. Hip Int 2012;22(04):397-402

4 Barquet A, Gelink A, Giannoudis PV. Proximal femoral fractures and vascular injuries in adults: Incidence, aetiology and outcomes. Injury 2015;46(12):2297-2313

5 Hamoui M, Larbi A, Bommart S, Fauré P, Largey A, Canovas F. False aneurysm of perforating branch of the profunda femoris artery following intertrochanteric fracture, a rare vascular complication: clinical, radiological features and management: case report and review of the literature. Eur J Orthop Surg Traumatol 2010; 20:59-65

6 Neubauer T, Grechenig S, Leitner L, Auffarth A, Plecko M. Vascular complications in plating of the proximal femur: review. Arch Orthop Trauma Surg 2016;136(04):539-551

7 Keel JD, Eyres KS. Vascular injury by an intertrochanteric fracture fragment. Injury 1993;24(05):350-352

8 Entwisle JJ, De Nunzio M, Hinwood D. Case report: Transcatheter embolization of pseudoaneurysm of the profunda femoris artery complicating fracture of the femoral neck. Clin Radiol 2001;56 (05):424-427

9 Pradhan DJ, Juanteguy JM, Wilder RJ, Michelson E. Arterial injuries of the extremities associated with fractures. Arch Surg 1972;105 (04):582-585

10 Hanna GB, Holdsworth RJ, McCollum PT. Profunda femoris artery pseudoaneurysm following orthopaedic procedures. Injury 1994; 25(07):477-479 